\title{
Safety and efficacy of repeated crosslinking assisted by transepithelial double-cycle iontophoresis in keratoconus progression after primary corneal crosslinking
}

\author{
Huping $\mathrm{Wu}^{1,2,3} \cdot$ Lan $^{1} \mathrm{Li}^{1} \cdot$ Shunrong Luo ${ }^{1,3} \cdot$ Xie Fang $^{1,3} \cdot$ Xumin Shang $^{1,3} \cdot$ Zhiwen Xie $^{1} \cdot$ Xianwen Xiao $^{1,3}$. \\ Huan $\mathrm{He}^{1,3} \cdot$ Zhirong Lin $\mathbb{1}^{1,2,3} \cdot$ Zuguo Liu ${ }^{1,2}$
}

Received: 17 August 2020 / Revised: 27 November 2020 / Accepted: 2 December 2020 / Published online: 7 January 2021

(c) The Author(s) 2021. This article is published with open access

\begin{abstract}
Objectives To evaluate the safety and efficacy of repeated corneal collagen crosslinking assisted by transepithelial doublecycle iontophoresis (DI-CXL) in the management of keratoconus progression after primary CXL.

Methods A retrospective analysis was conducted in the patients who underwent repeated CXL between 2016 and 2018. These patients were treated with DI-CXL if keratoconus progression was confirmed after primary CXL. Scoring of ocular pain and corneal epithelial damage, visual acuity, corneal tomography, in vivo corneal confocal microscopy (IVCM) was performed before and at 3, 6, 12, and 24 months after DI-CXL.

Results Overall, 21 eyes of 12 patients (mean age $17.3 \pm 1.9$ years) were included in this study. Before DI-CXL, an average increase of $4.26 \mathrm{D}$ in $K_{\max }$ was detected in these patients with a mean follow-up interval of $(23.0 \pm 13.7)$ months. After DICXL, corneal epithelial damage rapidly recovered within days. Visual acuity remained unchanged with follow-up of 24 months. When compared to baseline, significant decreases were observed in $K_{\max }$ (at 3 months) and K2 (at 3 and 6 months) after DI-CXL. Corneal thickness of thinnest point significantly decreased at 3 months postoperatively. When compared to baseline, no significant differences were found in any of the refractive or tomographic parameters at 12 and 24 months. IVCM revealed trabecular patterned hyperdense tissues after DI-CXL in the anterior stroma at the depth of 200 $\mu \mathrm{m}$ or more. No corneal infiltration or persistent epithelial defect was recorded after DI-CXL.

Conclusion DI-CXL is safe and effective as a good alternative in stabilizing keratoconus progression after primary CXL.
\end{abstract}

\section{Introduction}

Keratoconus is a progressive eye disease with degeneration and reduced biomechanical stability in the cornea. The loss of stability leads to protrusion and a subsequent

Zhirong Lin

charlie.lyn668@foxmail.com

$\triangle$ Zuguo Liu

zuguoliu@xmu.edu.cn

1 Eye Institute and Affiliated Xiamen Eye Center of Xiamen University, Fujian, Xiamen, China

2 Fujian Provincial Key Laboratory of Ophthalmology and Visual Science, Xiamen 361102 Fujian, China

3 Fujian Key Laboratory of Ocular Surface and Corneal Disease (affiliated Xiamen Eye Center of Xiamen University), Xiamen 361003 Fujian, China increase in stromal thinning, thus resulting in an irregular corneal astigmatism and impaired vision. In very severe cases, lamellar or penetrating corneal transplantation is the therapeutic option to regain vision. As a progressive corneal disease, keratoconus has a considerable impact on patient's vision and life quality. In recent years, corneal collagen crosslinking (CXL) has been recognized as a safe and effective treatment to delay or halt further progression of $\mathrm{KC}$ [1-3] and can reduce the need of keratoplasty $[4,5]$. During CXL, riboflavin interacts with ultraviolet A light to create crosslinking of protein fibrils followed by the formation of interchain disulfide bonds, thus arresting the progression of corneal ectasia by increasing the biomechanical stability of the cornea. Various protocols [6] of CXL have been performed and extensively investigated, showing long-term efficacy of stabilization and improvement for keratoconus. CXL has become one of the standard treatments of progressive keratoconus in the world. 
On the other hand, failure and progression of keratectasia after primary CXL have also been reported [7, 8]. Although the definition and criteria of progression after CXL has been reported [9], a series of studies [10-13] revealed diverse signs of deterioration after CXL, including tomographic progression, worse ocular refractive status, and even morphological alteration observed by anterior segment optical coherence tomography or in vivo confocal microscopy. Whether repeated CXL should be performed to manage keratoconus progression needs more elucidation. So far, very few studies evaluated the safety and efficacy of recrosslinking in keratoconus with progression after CXL. Joella et.al demonstrated [13] that repeated CXL using classic epi-off protocol may be safe and effective.

However, potential complication [14-16] using standard Dresden's protocol, such as corneal haze, sterile corneal infiltrates, recurrent erosion syndrome, etc. should be taken into consideration, as repeated epithelial removal may double the risk of postoperative complications theoretically. Hence, epi-on protocol might be a better choice for repeated crosslinking. Iontophoresis, in which an electrical gradient is used to drive negatively charged riboflavin molecules across the intact epithelium, may further enhance riboflavin penetration in transepithelial CXL. Laboratory [17-19] and clinical studies [20] of iontophoresis have been encouraging, demonstrating increased transepithelial penetration and improvement of corneal biomechanics. Nevertheless, most studies [21-23] showed inferior results of standard protocol of iontophoresis when compared to epi-off protocol. Theoretically, two continuous cycles of standard iontophoresis allowed time for riboflavin to penetrate and diffuse more posteriorly. Some study [24] showed better outcome of transepithelial CXL assisted by two continuous cycles of iontophoresis (enhanced iontophoresis) than that by standard iontophoresis. Hence, this study is aimed to investigate the safety and efficacy of transepithelial recrosslinking assisted by double-cycle iontophoresis (DI) in patients with keratoconus progression after a primary CXL, as well as the characteristics in visual acuity (VA), corneal tomography, and morphological alteration in the corneal stroma.

\section{Subjects and methods}

\section{Subjects and criteria}

A retrospective study was conducted in patients who underwent the first CXL at the Affiliated Xiamen Eye Center of Xiamen University. The progression of keratoconus after CXL was identified [25] based on the presence of two or more of the following criteria: increase in $K_{\max }$ value $(\geq 1 \mathrm{D})$ in the tomography map difference between two consecutive corneal tomographies over at least 6 months after the first CXL, a deterioration of VA defined as a drop of one or more lines, or any changes in the refractive astigmatism as a change of $1.0 \mathrm{D}$ or above.

Eyes with corneal thickness less than $380 \mu \mathrm{m}$ at the thinnest point were excluded. Written informed consent was obtained from patients themselves (for subjects above 18 years old) or from their parents (for subjects below 18 years old). The study and surgical protocol were both approved by the hospital's ethics committee and were performed according to the tenets of the Declaration of Helsinki.

\section{Surgical procedures}

For re-crosslinking, transepithelial CXL assisted by doublecycle iontophoresis CXL (DI-CXL) was performed under sterile conditions in the operating room. Topical $0.1 \%$ pilocarpine eye drops were instilled $30 \mathrm{~min}$ before surgery. Topical $0.5 \%$ proparacaine hydrochloride eye drops were instilled twice before surgery (every $5 \mathrm{~min}$ ). For DI-CXL, the return electrode was affixed to the skin of frontal region, while the corneal iontophoresis electrode was attached to the cornea by a vacuum adsorption device (SOOFT, Italy). The corneal electrode was filled with approximately $0.5 \mathrm{~mL}$ of $0.1 \%$ riboflavin solution (Ricrolin ${ }^{+}$, SOOFT, Italy), which was specifically designed for iontophoresis, from the open proximal side until the stainless-steel mesh was completely immersed. After that, the device was connected to a constant current generator (I-ON XL, SOOFT, Italy) set at $1 \mathrm{~mA}$ current. Continuous double cycles of iontophoresis were conducted without interval. The total dose of $10 \mathrm{~mA} /$ 10 min (enhanced iontophoresis, which is different from the standard dose [26] of $5 \mathrm{~mA} / 5 \mathrm{~min}$ ) was monitored by the generator.

After completion of enhanced iontophoresis, residual riboflavin was rinsed away. Ultraviolet A irradiation [27] of $9 \mathrm{~mW} /$ $\mathrm{cm}^{2}$ with a wavelength of $365 \mathrm{~nm}$ was initiated using the ultraviolet lamp system (KXL system, Avedro, USA) for 10 min using a continuous mode. The UV light was then focused on the apex of the cornea through the double red crosshair alignment laser system. During irradiation, drops of balanced solution were applied to the cornea every $1 \mathrm{~min}$ to keep moisture and rinse away residual riboflavin. Tobramycin and dexamethasone eye ointment (Alcon, Novartis, Switzerland) was applied to the conjunctival sac postoperatively. Subsequent treatment included $0.5 \%$ loteprednol and tobramycin eye drops four times per day and tapered over 4 weeks, topical artificial tears of $0.3 \%$ hyaluronate sodium four times per day for at least 8 weeks. No soft therapeutic contact lens was applied even when the epithelium damage was observed. 


\section{Evaluation of ocular discomfort and postoperative recovery}

The Numeric Rating Scale (NRS) system [28] was applied to capture information from the patient's perspective and the patients were asked to rate the severity of their symptom on a $0-10$ scale. When applied to ocular pain, 0 reflected no pain, and 10 reflected the worst possible pain. Higher scores indicated more severe symptom of ocular pain.

The bulbar conjunctival redness ranged from 0 to 4 was provided by the instrument (Keratograph $5 \mathrm{M}$, OCULUS, Wetzlar, Germany) through comparing the photos captured with standard pictures stored in the program. Higher scores indicated greater severity of bulbar conjunctival congestion [29].

The epithelial fluorescein staining score was graded with cobalt blue light by a masked observer. Pictures were taken with a digital camera (BQ900 with IM900 digital imaging module, Haag-Streit, Switzerland). The extent of corneal epithelial damage was scored [30] according to the following scale: 0 , no staining; 1 , slight punctate staining (less than 30 points); 2, diffuse punctate staining (more than 30 points but less than 100 points); 3 , diffuse staining more than 100 points; 4 , diffuse staining with plaque covering less than one third of the cornea; 5, diffuse staining with plaque covering more than one third but less than two third of the cornea; and 6 , staining with huge plaque covering more than two thirds of the cornea.

\section{Ocular examinations}

In the preoperative and postoperative examinations, the following parameters were accessed: uncorrected distance visual acuity (UCVA), best corrected distance visual acuity (BCVA), slit-lamp microscopy examination including corneal fluorescein sodium staining, corneal tomography and pachymetry (Pentacam HR 70900, Oculus, Wetzlar, Germany), endothelial biomicroscope (SP-3000P, Topcon, Tokyo, Japan), in vivo corneal confocal microscopy (IVCM, HRT3/Rostock Cornea Module, Heidelberg Engineering $\mathrm{GmbH}$, Germany). Keratometric values ( $K_{\max }, \mathrm{K} 1$ and $\mathrm{K} 2$ ), minimum pachymetry values, etc. were derived from the tomography data. All patients were assessed at baseline and followed up for at least 24 months postoperatively.

\section{Statistical analysis}

The data was imported to the Statistical Package for Social Sciences (SPSS Inc., Chicago, IL, version 16.0) for analysis. Repeated measures one-way ANOVA was used for statistical comparisons among timepoints. Bonferroni correction was made for multiple comparisons. The significance level was set at $<0.05$.

\section{Results}

\section{Demographics of the subjects}

A total of 625 patients (1027 eyes) with keratoconus were treated with a primary CXL procedure in our hospital from 2011 to 2017. During follow-up, 498 patients adhered to follow-up. Of these 498 patients, 12 patients ( $2.41 \%$, eight male and four female) were diagnosed as having keratoconus progression after primary CXL. These patients (21 eyes in total) with keratoconus progression after primary CXL were included in this study and received a repeated CXL procedure between July 2016 and June 2018. Demographics of patients were listed in Table 1. The mean age of the patients was $(17.3 \pm 1.9)$ years. Before re-CXL, an average increase of $4.26 \mathrm{D}$ in $K_{\max }$ was detected in these patients with a mean follow-up interval of $(23.0 \pm 13.7)$ months. After re-crosslinking, all of these patients attended the follow-up visit for at least 24 months.

\section{Slit-lamp observation and ocular discomfort}

Before surgery, no positive fluorescein staining or few punctual staining was found in the cornea. Moderate corneal epithelial fluorescein staining score and slight to moderate NRS score was observed on day 1 and 3 postoperatively, and decreased to a very low level after day 7 (Fig. 1a, b). Conjunctival congestion was apparently increased on day 1 and 3 postoperative and almost disappeared after day 7 (Fig. 1c). Representative images of corneal epithelial defect are also shown (Fig. 1d-g).

\section{Refractive and tomographic changes after re-crosslinking}

Comparative analysis of the UCVA and BCVA as well as refractive parameters are shown in Table 2. After re-crosslinking, UCVA and BCVA slightly improved, however, no statistical difference was found when compared with the baseline. No significant difference was found in spherical dioptre, cylinder dioptre, or the spherical equivalent $(P>$ $0.05)$, although the three parameters were slightly decreased after re-CXL.

Corneal flattening was seen with significant decreases in $K_{\max }$ (at 3 months, $P=0.027$ ) and $\mathrm{K} 2$ (at 3 and 6 months, $P=0.022$ and $P=0.036$, respectively) after DI-CXL. Corneal thickness of thinnest point significantly decreased at 3 months postoperatively $(P<0.001)$. When compared to baseline, no significant differences were found in any of the refractive or tomographic parameters at 12 and 24 months after DI-CXL, indicating that the keratoconus had been stabilized during 24 months of follow-up. 
Table 1 Demographics of the patients before transepithelial re-crosslinking.

\begin{tabular}{|c|c|c|c|c|c|c|c|}
\hline Patient & Sex & Age, years & Eye & $\begin{array}{l}\text { Time of progression after primary } \\
\text { CXL, months }\end{array}$ & $\begin{array}{l}\text { Increase in } \\
K_{\max }, \mathrm{D}\end{array}$ & Protocol of Primary CXL & $\mathrm{AC}$ \\
\hline \multirow[t]{2}{*}{1} & M & 15 & $\mathrm{OD}$ & 11 & 4.5 & Standard iontophoresis, $9 \mathrm{~mW} / \mathrm{cm}^{2}, 10 \mathrm{~min}$ & $\mathrm{Y}$ \\
\hline & & & OS & 11 & 2.3 & & \\
\hline 2 & $\mathrm{~F}$ & 19 & OS & 54 & 8.8 & Epi-off, $9 \mathrm{~mW} / \mathrm{cm}^{2}, 10 \mathrm{~min}$ & $\mathrm{Y}$ \\
\hline \multirow[t]{2}{*}{3} & $\mathrm{~F}$ & 18 & OD & 48 & 13.0 & Standard iontophoresis, $9 \mathrm{~mW} / \mathrm{cm}^{2}, 10 \mathrm{~min}$ & Y \\
\hline & & & OS & 48 & 12.1 & & \\
\hline 4 & M & 17 & OS & 9 & 5.2 & $\begin{array}{l}\text { Transepithelial KXL system, } 45 \mathrm{~mW} / \mathrm{cm}^{2}, 5 \\
\text { min and } 20 \mathrm{~s}\end{array}$ & $\mathrm{Y}$ \\
\hline \multirow[t]{2}{*}{5} & M & 17 & OD & 16 & 3.8 & Standard iontophoresis, $9 \mathrm{~mW} / \mathrm{cm}^{2}, 10 \mathrm{~min}$ & $\mathrm{Y}$ \\
\hline & & & OS & 16 & 2.6 & & \\
\hline \multirow[t]{2}{*}{6} & M & 16 & OD & 16 & 5.5 & $\begin{array}{l}\text { Transepithelial KXL system, } 45 \mathrm{~mW} / \mathrm{cm}^{2}, 5 \\
\text { min and } 20 \mathrm{~s}\end{array}$ & $\mathrm{Y}$ \\
\hline & & & OS & 16 & 3.5 & & \\
\hline \multirow[t]{2}{*}{7} & M & 17 & $\mathrm{OD}$ & 22 & 4.7 & Standard iontophoresis, $9 \mathrm{~mW} / \mathrm{cm}^{2}, 10 \mathrm{~min}$ & $\mathrm{~N}$ \\
\hline & & & OS & 22 & 2.1 & & \\
\hline \multirow[t]{2}{*}{8} & $\mathrm{~F}$ & 15 & OD & 12 & 2.5 & $\begin{array}{l}\text { Transepithelial KXL system, } 45 \mathrm{~mW} / \mathrm{cm}^{2}, 5 \\
\text { min and } 20 \mathrm{~s}\end{array}$ & $\mathrm{Y}$ \\
\hline & & & OS & 12 & 2.2 & & \\
\hline 9 & $\mathrm{~F}$ & 20 & OS & 27 & 3.3 & Epi-off, $9 \mathrm{~mW} / \mathrm{cm}^{2}, 10 \mathrm{~min}$ & $\mathrm{Y}$ \\
\hline \multirow[t]{2}{*}{10} & M & 16 & OD & 19 & 1.9 & $\begin{array}{l}\text { Transepithelial KXL system, } 45 \mathrm{~mW} / \mathrm{cm}^{2}, 5 \\
\text { min and } 20 \mathrm{~s}\end{array}$ & $\mathrm{Y}$ \\
\hline & & & OS & 19 & 1.3 & & \\
\hline \multirow[t]{2}{*}{11} & M & 21 & OD & 38 & 3.9 & Epi-off, $9 \mathrm{~mW} / \mathrm{cm}^{2}, 10 \mathrm{~min}$ & $\mathrm{~N}$ \\
\hline & & & OS & 38 & 1.9 & & \\
\hline \multirow[t]{2}{*}{12} & M & 16 & OD & 15 & 1.7 & $\begin{array}{l}\text { Transepithelial KXL system, } 45 \mathrm{~mW} / \mathrm{cm}^{2}, 5 \\
\text { min and } 20 \mathrm{~s}\end{array}$ & $\mathrm{Y}$ \\
\hline & & & OS & 15 & 2.6 & & \\
\hline
\end{tabular}

$C X L$ corneal collagen crosslinking, $A C$ allergic conjunctivitis.
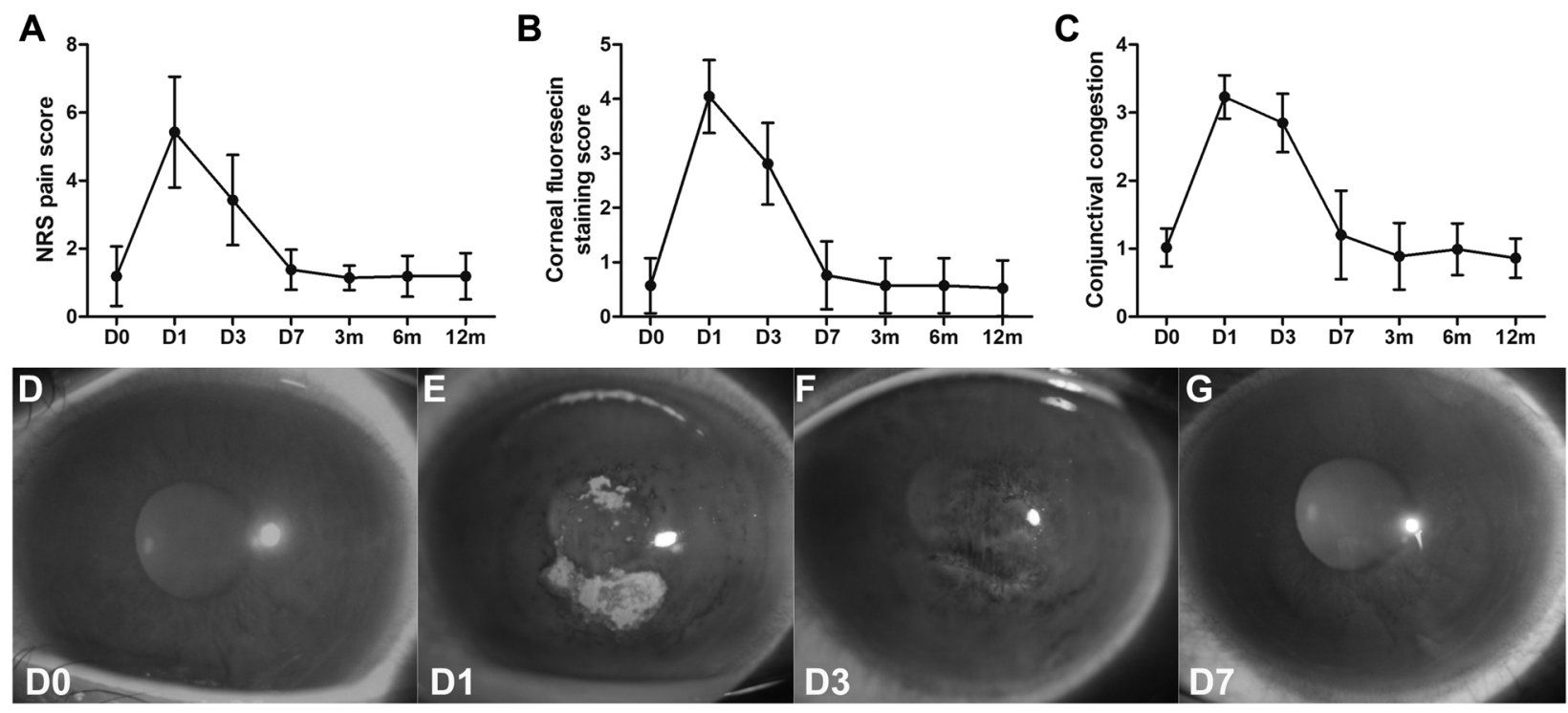

Fig. 1 Ocular discomfort and slit lamp observation after repeated CXL. Line charts showed the NRS pain score (a), corneal fluorescein sodium staining score (b), and the conjunctival congestion score (c) before and after transepithelial re-crosslinking. Representative image showing the epithelial defect and recovery after re-crosslinking in one patient (d-g). On day 1 after re-crosslinking (e), both small plaque-like and punctual epithelial staining was seen within the central cornea (9 $\mathrm{mm}$ diameter). 
Table 2 Refractive and tomographic changes after transepithelial re-crosslinking.

\begin{tabular}{|c|c|c|c|c|c|c|c|c|c|}
\hline & Before re-crosslinking & 3 months & & 6 months & & 12 months & & 24 months & \\
\hline$(n=21)$ & Mean \pm SD & Mean \pm SD & $p$ & Mean \pm SD & $p$ & Mean \pm SD & $p$ & Mean \pm SD & $p$ \\
\hline UCVA & $1.02 \pm 0.32$ & $1.03 \pm 0.32$ & 1.000 & $1.07 \pm 0.38$ & 0.524 & $1.04 \pm 0.35$ & 1.000 & $1.03 \pm 0.36$ & 1.000 \\
\hline BCVA & $0.34 \pm 0.09$ & $0.31 \pm 0.11$ & 1.000 & $0.32 \pm 0.13$ & 1.000 & $0.36 \pm 0.14$ & 1.000 & $0.34 \pm 0.13$ & 1.000 \\
\hline Sphere, D & $-3.86 \pm 2.79$ & $-3.46 \pm 1.93$ & 1.000 & $-3.58 \pm 2.17$ & 1.000 & $-3.63 \pm 2.35$ & 1.000 & $-3.61 \pm 2.33$ & 1.000 \\
\hline Cylinder, D & $-3.48 \pm 1.67$ & $-2.74 \pm 1.36$ & 0.178 & $-2.82 \pm 1.42$ & 0.205 & $-3.04 \pm 1.19$ & 0.984 & $-2.98 \pm 1.17$ & 0.654 \\
\hline SE, D & $-5.60 \pm 2.98$ & $-4,83 \pm 2.01$ & 0.619 & $-4.99 \pm 2.29$ & 0.629 & $-5.15 \pm 2.48$ & 1.000 & $-5.10 \pm 2.48$ & 1.000 \\
\hline $\mathrm{K} 1, \mathrm{D}$ & $52.25 \pm 4.98$ & $52.00 \pm 4.93$ & 0.389 & $52.20 \pm 5.18$ & 1.000 & $52.39 \pm 5.40$ & 1.000 & $52.36 \pm 5.40$ & 1.000 \\
\hline $\mathrm{K} 2$, D & $56.87 \pm 4.56$ & $56.20 \pm 4.82$ & 0.022 & $56.21 \pm 4.90$ & 0.036 & $56.55 \pm 5.16$ & 1.000 & $56.50 \pm 5.17$ & 1.000 \\
\hline$K_{\max }, \mathrm{D}$ & $63.48 \pm 6.31$ & $62.45 \pm 6.86$ & 0.027 & $62.44 \pm 6.80$ & 0.179 & $62.59 \pm 7.59$ & 1.000 & $62.59 \pm 7.56$ & 1.000 \\
\hline Minimal thickness, $\mu \mathrm{m}$ & $409.9 \pm 29.7$ & $390.4 \pm 32.1$ & $<0.001$ & $404.3 \pm 28.5$ & 0.085 & $405.5 \pm 26.9$ & 0.105 & $405.0 \pm 27.4$ & 0.067 \\
\hline $\mathrm{EC}$, cells $/ \mathrm{mm}^{2}$ & $2668 \pm 220.0$ & $2642 \pm 177.6$ & 1.000 & $2641 \pm 188.7$ & 1.000 & $2644 \pm 192.2$ & 1.000 & $2644 \pm 187.1$ & 1.000 \\
\hline $\mathrm{IOP}, \mathrm{mm} \mathrm{Hg}$ & $13.42 \pm 2.03$ & $14.00 \pm 2.42$ & 1.000 & $13.49 \pm 1.64$ & 1.000 & $13.39 \pm 1.92$ & 1.000 & $13.45 \pm 1.88$ & 1.000 \\
\hline
\end{tabular}

$U C V A$ uncorrected visual acuity, $B C V A$ best corrected visual acuity, $S E$ spherical equivalent, $E C$ endothelial cell, $I O P$ intraocular ocular pressure.

\section{Structural alteration in the corneal stroma}

IVCM was assessed to evaluate the structural alternation in the corneal stroma. Representative images are shown in Fig. 2.

\section{Postoperative corneal complications}

No significant endothelial cell loss or IOP elevation after DI-CXL was found. Corneal complications including corneal haze, sterile corneal infiltrates, recurrent epithelium erosion, corneal melting or perforation were not noted during the period of follow-up.

\section{Discussion}

Long-term stability of progressive keratoconus after CXL treatment with even more than 10 years of follow-up has been shown in many studies, in which [2, 3, 31] some failures of CXL were also reported. So far, how to treat the patients with progression after a primary CXL is not extensively discussed, at least not systemically. Hafezi et al. [10] reported a repeated CXL procedure 4 years after the first CXL in one patient, and recorded a flattening effect in the cornea. Joella et al. [13] demonstrated that repeated CXL using the classic epi-off protocol, the same method in the primary CXL, might be safe and effective. Our results further support the concept that transepithelial recrosslinking assisted by double-cycle iontophoresis is effective and safe in the treatment of keratoconus progression after primary CXL.

In experimental studies, Beshtawi et al. [32] found that human corneas receiving 2 or 3 consecutive CXL treatments within $24 \mathrm{~h}$ had some increase in corneal stiffness but no statistically significant difference was found when compared with the single CXL treatment group. Tabibian et al. [33] reported that the stiffness of mouse cornea was not increased after a repeated CXL performed 3 days after the first CXL. These data indicated that no more crosslinks in the anterior stroma were induced by repeating CXL within a very short period. Our IVCM results showed that crosslinks in the stroma could be further induced by a repeated procedure after several months or years. Unfortunately, the optimal surgical time of repeated CXL remains unclear. In addition to deterioration of refractive and tomographic data, IVCM image showing the decrease of crosslinks might be considered as one of the indications of repeating CXL. However, the role of IVCM in the diagnosis and treatment of keratoconus progression needs further investigation [34].

Due to the limited number of recruited subjects, it was difficult to compare the effect of different protocols (epi-on vs. epi-off, etc.) of repeated CXL. Our data showed an improvement of tomographic reading including $K_{\max }$ and $\mathrm{K} 2$ at 3 and 6 months after DI-CXL. However, at 12 and 24 months after re-crosslinking, no statistically significant difference was found when compared with the baseline. This result might indicate that the corneal remodeling at 12 and 24 months after repeated CXL was being weakened. One possible reason for the weakening of crosslinks was that CXL effect by epi-on protocol was not as good as that by epi-off protocol [35], although the penetration depth of DI-CXL could be $250 \mu \mathrm{m}$, which was close to that in classic Dresden's protocol. Another reason might be that most of the included subjects were "advanced" keratoconus with progression, as the $K_{\max }$ values before re-CXL were greater than $58.0 \mathrm{D}$ in 15 eyes $(71.4 \%)$ in our study.

The limitations of epi-on CXL using iontophoresis were numerous and have been partially overcome by various modified protocols, some of which have shown better efficacy. The first limitation of iontophoresis was the fluence 


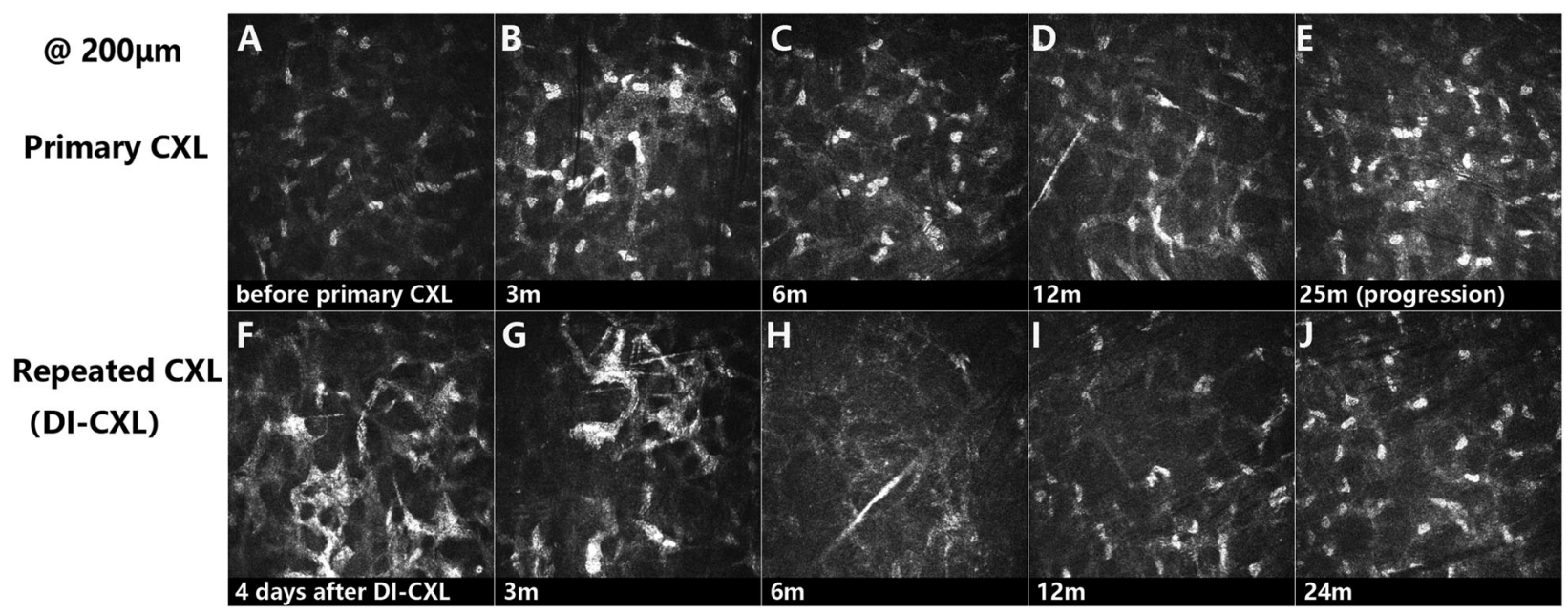

Fig. 2 Representative images showing the structural alteration in the corneal stroma by in vivo confocal microscopy $(800 \times)$. Different to the preoperative scan (a), lacunar oedema was visible in the first three postoperative months after primary CXL with trabecular patterned hyperdense tissue surrounding oedematous areas (b). After that, anterior-mid stroma was repopulated by keratocytes and surrounded by extracellular collagen tissue with slightly high density (c, d). Before re-crosslinking, hyper-reflective extracellular tissue surrounding keratocyte nuclei could hardly be seen in the anterior stroma

according to epithelial photo-attenuation or absorption of UV-A energy at $370 \mathrm{~nm}$ waveband. This could be compensated [36] by enhancing the fluence. The second main limitation of the iontophoresis was the oxygen consumption by the epithelium in situ limiting intraoperative oxygen diffusion. This could be compensated by using the pulsed light to UV exposure [37]. Recently, a new protocol which enhancing the fluence and application of pulsed light of UV exposure was reported and showed great outcome [38, 39]. Our results also showed double-cycle iontophoresis was effective and safe in the treatment of keratoconus progression. However, there might be limitations for a double cycle of iontophoresis that increased riboflavin concentration further limiting the oxygen diffusion and UV-A absorption and increasing the water content into the stroma thus leading to hypotony. Theoretically, high water content and stromal oedema after double iontophoresis increase the distance between collagen fibrils thus reducing crosslinks formations. However, increased corneal crosslinks and depth of crosslinking up to $25 \mu \mathrm{m}$ were still observed by IVCM in our study.

In the past decade, the risk factors associated with primary keratoconus have been extensively discussed in plenty of studies [40-43]. However, the risk factors associated with keratoconus progression after a primary CXL remain unclear. Theoretically, the risk factors under the two conditions are comparable, such as eye rubbing [13], $K_{\max }$ higher than 58.0 D [11], and young age [12], etc. In our study, the history of allergic conjunctivitis was confirmed in 10 patients (83.3\%) who had a habit of eye rubbing. Before re-CXL, nine eyes

of these patients (e). After re-crosslinking, lacunar oedema in the anterior stroma reappeared and could be observed during the early period postoperatively (f). The depth of cornea oedema could still be observed at $250 \mu \mathrm{m}$ measured from epithelial surface. Apoptotic keratocytes and activated keratocytes with elongated membrane processes were both detectable from 3 to 6 months postoperatively $(\mathbf{g}, \mathbf{h})$. At 12 and 24 months after re-CXL, anterior-mid stroma was repopulated by keratocytes and surrounded by dense extracellular collagen tissue (i, j).

(42.9\%) in five patients had $K_{\max }$ higher than $58.0 \mathrm{D}$ and lower than $70.0 \mathrm{D}$, while six eyes $(28.6 \%)$ in three patients represented $K_{\max }$ higher than 70.0 D. Eight patients $(66.7 \%)$ in our study were younger than 18 years old. Therefore, patients with these risk factors need close follow-up.

\section{Conclusions}

In conclusion, transepithelial re-crosslinking assisted by double-cycle iontophoresis could be considered as a good alternative with high safety and efficacy in stabilizing keratoconus progression after a primary CXL. However, the long-term effects of this protocol need further study.

\section{Summary table}

\section{What was known before}

- Keratoconus progression after primary corneal crosslinking could be observed and repeating corneal crosslinking could be one of the solutions.

- However, repeated crosslinking procedure could increase the risk of complications.

\section{What this study adds}

- Transepithelial corneal crosslinking with double-cycle iontophoresis is an effective and safe protocol in 
stabilizing keratoconus progression after primary corneal crosslinking surgery.

\section{Data availability}

The datasets generated and/or analyzed during the present study are not publicly available (obtained from the affiliated Xiamen Eye Center of Xiamen University, Xiamen repository), but are available from the corresponding author upon reasonable request.

Funding This research was supported by grants from the National Natural Science Foundation of China (81570816,81570815), Fujian Provincial Science Fund for Distinguished Young Scholars (2020D029), Xiamen Science and Technology Program for Public Wellbeing (3502Z20174003), Xiamen Medical and Health Project (3502Z20189024), Huaxia Translational Medicine Fund for Young Scholars (2018-A-006), Research Project of Health and Family Planning for Youth in Fujian Province (2017-2-117, 2018-2-79). The funding sources had no role in the design and conduct of the study; collection, analysis and interpretation of the data; preparation, review, approval, and submission of the manuscript.

Author contributions ZL and ZL conceived the study. HW, LL, SL, XF, XS, ZX, XX, and HH collected the data. HW and LL did the statistical analysis of the data. LL and XF further analyzed the data. HW and LL prepared the manuscript. All the authors contributed to the manuscript and approved the final manuscript for submission.

\section{Compliance with ethical standards}

Conflict of interest The authors declare that they have no conflict of interest.

Publisher's note Springer Nature remains neutral with regard to jurisdictional claims in published maps and institutional affiliations.

Open Access This article is licensed under a Creative Commons Attribution 4.0 International License, which permits use, sharing, adaptation, distribution and reproduction in any medium or format, as long as you give appropriate credit to the original author(s) and the source, provide a link to the Creative Commons license, and indicate if changes were made. The images or other third party material in this article are included in the article's Creative Commons license, unless indicated otherwise in a credit line to the material. If material is not included in the article's Creative Commons license and your intended use is not permitted by statutory regulation or exceeds the permitted use, you will need to obtain permission directly from the copyright holder. To view a copy of this license, visit http://creativecommons. org/licenses/by/4.0/.

\section{References}

1. Wollensak G, Spoerl E, Seiler T. Riboflavin/ultraviolet-a-induced collagen crosslinking for the treatment of keratoconus. Am J Ophthalmol. 2003;135:620-7.

2. Raiskup F, Theuring A, Pillunat LE, Spoerl E. Corneal collagen crosslinking with riboflavin and ultraviolet-A light in progressive keratoconus: ten-year results. J Cataract Refract Surg. 2015;41:41-6.

3. Mazzotta C, Traversi C, Baiocchi S, Bagaglia S, Caporossi O, Villano A, et al. Corneal collagen cross-linking with riboflavin and ultraviolet A light for pediatric keratoconus: ten-year results. Cornea 2018;37:560-6.

4. Godefrooij DA, Gans R, Imhof SM, Wisse RP. Nationwide reduction in the number of corneal transplantations for keratoconus following the implementation of cross-linking. Acta Ophthalmol. 2016;94:675-8.

5. Sandvik GF, Thorsrud A, Råen M, Østern AE, Sæthre M, Drolsum L. Does corneal collagen cross-linking reduce the need for keratoplasties in patients with keratoconus? Cornea. 2015;34:991-5.

6. O’Brart DPS. Corneal collagen crosslinking for corneal ectasias: a review. Eur J Ophthalmol. 2017;27:253-69.

7. Koller T, Mrochen M, Seiler T. Complication and failure rates after corneal crosslinking. J Cataract Refract Surg. 2009;35: 1358-62.

8. Caporossi A, Mazzotta C, Paradiso AL, Baiocchi S, Marigliani D, Caporossi T. Transepithelial corneal collagen crosslinking for progressive keratoconus: 24-month clinical results. J Cataract Refract Surg. 2013;39:1157-63.

9. Gomes JA, Tan D, Rapuano CJ, Belin MW, Ambrósio R Jr., Guell $\mathrm{JL}$, et al. Global consensus on keratoconus and ectatic diseases. Cornea 2015;34:359-69.

10. Hafezi F, Tabibian D, Richoz O. Additive effect of repeated corneal collagen cross-linking in keratoconus. J Refract Surg. 2014;30:716-8.

11. Kuechler SJ, Tappeiner C, Epstein D, Frueh BE. Keratoconus progression after corneal cross-linking in eyes with preoperative maximum keratometry values of 58 diopters and steeper. Cornea. 2018;37:1444-8.

12. Chatzis N, Hafezi F. Progression of keratoconus and efficacy of pediatric [corrected] corneal collagen cross-linking in children and adolescents. J Refract Surg. 2012;28:753-8.

13. Antoun J, Slim E, El Hachem R, Chelala E, Jabbour E, Cherfan G, et al. Rate of corneal collagen crosslinking redo in private practice: risk factors and safety. J Ophthalmol. 2015;2015:690961.

14. Dhawan S, Rao K, Natrajan S. Complications of corneal collagen cross-linking. J Ophthalmol. 2011;2011:869015.

15. Lam FC, Geourgoudis P, Nanavaty MA, Khan S, Lake D. Sterile keratitis after combined riboflavin-UVA corneal collagen crosslinking for keratoconus. Eye. 2014;28:1297-303.

16. Evangelista CB, Hatch KM. Corneal collagen cross-linking complications. Semin Ophthalmol. 2018;33:29-35.

17. Lombardo M, Serrao S, Rosati M, Ducoli P, Lombardo G. Biomechanical changes in the human cornea after transepithelial corneal crosslinking using iontophoresis. J Cataract Refract Surg. 2014;40:1706-15

18. Gore DM, O'Brart D, French P, Dunsby C, Allan BD. Transepithelial riboflavin absorption in an ex vivo rabbit corneal model. Invest Ophthalmol Vis Sci. 2015;56:5006-11.

19. Cassagne M, Laurent C, Rodrigues M, Galinier A, Spoerl E, Galiacy SD, et al. Iontophoresis transcorneal delivery technique for transepithelial corneal collagen crosslinking with riboflavin in a rabbit model. Invest Ophthalmol Vis Sci. 2016;57:594-603.

20. Buzzonetti L, Petrocelli G, Valente P, Iarossi G, Ardia R, Petroni S. Iontophoretic transepithelial corneal cross-linking to halt keratoconus in pediatric cases: 15-month follow-up. Cornea. 2015;34:512-5.

21. Bouheraoua N, Jouve L, El Sanharawi M, Sandali O, Temstet C, Loriaut $\mathrm{P}$, et al. Optical coherence tomography and confocal microscopy following three different protocols of corneal collagen-crosslinking in keratoconus. Invest Ophthalmol Vis Sci. 2014;55:7601-9. 
22. Buzzonetti L, Petrocelli G, Valente P, Iarossi G, Ardia R, Petroni $\mathrm{S}$, et al. Iontophoretic transepithelial collagen cross-linking versus epithelium-off collagen cross-linking in pediatric patients: 3-year follow-up. Cornea 2019;38:859-63.

23. Cerman E, Toker E, Ozarslan Ozcan D. Transepithelial versus epithelium-off crosslinking in adults with progressive keratoconus. J Cataract Refract Surg. 2015;41:1416-25.

24. Liao K, Hu M, Chen F, Li P, Song P, Zeng QY. Clinical and microstructural changes with different iontophoresis-assisted corneal cross-linking methods for keratoconus. Int $\mathrm{J}$ Ophthalmol. 2019;12:219-25.

25. Wang YM, Chan TC, Yu MCY, Jhanji V. Comparative evaluation of progression rate in keratoconus before and after collagen crosslinking. Br J Ophthalmol. 2018;102:1109-13.

26. Bikbova G, Bikbov M. Transepithelial corneal collagen crosslinking by iontophoresis of riboflavin. Acta Ophthalmol. 2014;92: e30-4.

27. Ng AL, Chan TC, Cheng AC. Conventional versus accelerated corneal collagen cross-linking in the treatment of keratoconus. Clin Exp Ophthalmol. 2016;44:8-14.

28. Karcioglu O, Topacoglu H, Dikme O, Dikme O. A systematic review of the pain scales in adults: which to use? Am J Emerg Med. 2018;36:707-14.

29. Downie LE, Keller PR, Vingrys AJ. Assessing ocular bulbar redness: a comparison of methods. Ophthalmic physiological Opt: J Br Coll Ophthalmic Opticians. 2016;36:132-9.

30. Pauly A, Brignole-Baudouin F, Labbe A, Liang H, Warnet JM, Baudouin C. New tools for the evaluation of toxic ocular surface changes in the rat. Invest Ophthalmol Vis Sci. 2007;48:5473-83.

31. Hashemi H, Seyedian MA, Miraftab M, Fotouhi A, Asgari S. Corneal collagen cross-linking with riboflavin and ultraviolet a irradiation for keratoconus: long-term results. Ophthalmology. 2013;120:1515-20.

32. Beshtawi IM, Akhtar R, Hillarby MC, O'Donnell C, Zhao X, Brahma A, et al. Biomechanical changes after repeated collagen cross-linking on human corneas assessed in vitro using scanning acoustic microscopy. Invest Ophthalmol Vis Sci. 2014;55:1549-54.
33. Tabibian D, Kling S, Hammer A, Richoz O, Hafezi F. Repeated cross-linking after a short time does not provide any additional biomechanical stiffness in the mouse cornea in vivo. J Refract Surg. 2017;33:56-60.

34. Mazzotta C, Hafezi F, Kymionis G, Caragiuli S, Jacob S, Traversi $\mathrm{C}$, et al. In vivo confocal microscopy after corneal collagen crosslinking. Ocul Surf. 2015;13:298-314.

35. Kobashi H, Rong SS, Ciolino JB. Transepithelial versus epithelium-off corneal crosslinking for corneal ectasia. J Cataract Refract Surg. 2018;44:1507-16.

36. Kolozsvári L, Nógrádi A, Hopp B, Bor ZUV. absorbance of the human cornea in the 240- to 400-nm range. Invest Ophthalmol Vis Sci. 2002;43:2165-8.

37. Torres-Netto EA, Kling S, Hafezi N, Vinciguerra P, Randleman JB, Hafezi F. Oxygen diffusion may limit the biomechanical effectiveness of iontophoresis-assisted transepithelial corneal cross-linking. J Refract Surg. 2018;34:768-74.

38. Mazzotta C, Bagaglia SA, Sgheri A, Di Maggio A, Fruschelli M, Romani A, et al. Iontophoresis corneal cross-linking with enhanced fluence and pulsed UV-A light: 3-year clinical results. J Refract Surg. 2020;36:286-92.

39. Mazzotta C, Bagaglia SA, Vinciguerra R, Ferrise M, Vinciguerra P. Enhanced-fluence pulsed-light iontophoresis corneal crosslinking: 1-year morphological and clinical results. J Refract Surg. 2018;34:438-44.

40. Merdler I, Hassidim A, Sorkin N, Shapira S, Gronovich Y, Korach Z. Keratoconus and allergic diseases among Israeli adolescents between 2005 and 2013. Cornea. 2015;34:525-9.

41. Galvis V, Sherwin T, Tello A, Merayo J, Barrera R, Acera A. Keratoconus: an inflammatory disorder? Eye. 2015;29: 843-59.

42. Najmi H, Mobarki Y, Mania K, Altowairqi B, Basehi M, Mahfouz MS, et al. The correlation between keratoconus and eye rubbing: a review. Int J Ophthalmol. 2019;12:1775-81.

43. Lee HK, Jung EH, Cho BJ. Epidemiological association between systemic diseases and keratoconus in a korean population: a 10year nationwide cohort study. Cornea. 2020;3:348-53. 